\title{
Prolonged Tp-e interval and Tp-e/QT correlates well with modified Rodnan skin severity score in patients with systemic sclerosis
}

\author{
Sercan Okutucu ${ }^{1}$, Ugur Nadir Karakulak², Hakan Aksoy ${ }^{1}$, Cengiz Sabanoglu \\ Vedat Hekimsoy ${ }^{4}$, Levent Sahiner ${ }^{4}$, Ergun Baris Kaya ${ }^{4}$, Ali Akdogan, \\ Giray Kabakci ${ }^{4}$, Kudret Aytemir ${ }^{4}$, Lale Tokgozoglu ${ }^{4}$, Ali Oto ${ }^{1}$ \\ ${ }^{1}$ Department of Cardiology, Memorial Ankara Hospital, Ankara, Turkey \\ ${ }^{2}$ Department of Cardiology, Occupational Diseases Hospital, Ankara Turkey \\ ${ }^{3}$ Department of Cardiology, Kirikkale High Speciality Hospital, Kirikkale, Turkey \\ ${ }^{4}$ Department of Cardiology, Hacettepe University Faculty of Medicine, Ankara, Turkey
}

\begin{abstract}
Background: Ventricular arrhythmias can be seen in systemic sclerosis (SSc) patients and are thought to be a result of fibrosis or ischemia of the ventricular myocardium. Tp-e interval and Tp-e/QT ratio are electrocardiographic (ECG) indices to predict ventricular tachyarrhythmia and cardiovascular mortality. We aimed to evaluate Tp-e interval and Tp-e/QT ratio in patients with SSc.

Methods: A total of 107 patients with SSc (mean age, $48.6 \pm 14.0$ years; 96 females) and 100 healthy controls (mean age, $49.4 \pm 8.6$ years; 90 females) were enrolled. The standard 12-lead ECG was recorded; QTc, Tp-e interval and Tp-e/QT ratio were measured. Modified Rodnan skin severity score (MR-SSS) calculated for all SSc patients.

Results: Tp-e interval (90.7 $\pm 23.8 \mathrm{~ms}$ vs. $84.0 \pm 20.6 \mathrm{~ms}, p=0.032)$ and $T p-e / Q T$ ratio $(0.20 \pm 0.05$ vs. $0.18 \pm 0.04, p=0.007$, respectively) were significantly prolonged in $S S c$ patients than in the control group. Pearson's correlation analyses revealed positive correlations of MR-SSS with QTc $(r=0.427, p=0.001)$, Tp-e interval $(r=0.620, p=0.001)$ and Tp-el $/ Q T$ ratio $(r=0.615, p=0.001)$. MR-SSS $(\beta=2.108, p=0.001)$ and $C R P(\beta=2.273$, $p=0.027)$ were found to be significant independent predictors of Tp-e interval. Similarly, MR-SSS $(\beta=0.004, p=0.001)$ was only a significant independent predictor of Tp-e/QT ratio among patients with SSc.

Conclusions: The patients with SSc had a prolonged Tp-e interval and Tp-e/QT ratio compared with normal subjects. Furthermore, this prolongation was well correlated with clinical severity score among patients with SSc. Ventricular repolarization dispersion as a predictor of ventricular arrhythmias was found to be diminished in patients with SSc. Patients with SSc, particularly with higher MR-SSS, should be followed closely for adverse cardiovascular outcomes. (Cardiol J 2016; 23, 3: 242-249)
\end{abstract}

Key words: systemic sclerosis, Tp-e interval, Tp-e/QT ratio

Address for correspondence: Sercan Okutucu, MD, Memorial Ankara Hospital, Department of Cardiology, Cankaya, Ankara, P.O: 06520, Turkey, tel: +90 3122536666 (ext. 4207), fax: +90 312 2536623, e-mail: sercanokutucu@yahoo.com

Received: 17.01.2016

Accepted: 07.05.2016 


\section{Introduction}

Systemic sclerosis (SSc) is a systemic, inflammatory and autoimmune disease characterized by dysfunction of the endothelium, dysregulation of fibroblasts resulting in excessive production of collagen, and abnormalities of the immune system [1]. Even if presenting in a limited or diffuse form, progressive fibrotic process which is a pathologic feature of the disease, tends to involve the skin and multiple organ systems such as the gastrointestinal tract, kidneys and lungs [2]. The heart is also one of the major organs involved in SSc and the presence of cardiac involvement generally foreshadows poor prognosis. Cardiac involvement may be in the form of myositis, cardiac failure, cardiac fibrosis, coronary artery disease, conduction system abnormalities, and pericardial disease [3]. Depending on the degree of involvement, increased frequency of ventricular ectopic beats, as well as episodes of ventricular tachycardia can be seen in SSc patients and are thought to be a result of fibrosis or ischemia of the ventricular myocardium $[4,5]$.

Amplification of dispersion of ventricular repolarization has long been known as a substrate for ventricular arrhythmias and it has been evaluated by various methods, such as QT dispersion [6]. Recent studies indicate that Tp-e interval, which is the interval between the peak and end of the T wave on an electrocardiogram (ECG), can be used as an index of total (transmural, apico-basal and/or global) dispersion of repolarization [7]. Besides, increased Tp-e interval may also be a useful index to predict ventricular tachyarrhythmia and cardiovascular mortality $[8,9]$. Similarly, abnormally long or short QT interval is characterized by an increased propensity to develop ventricular tachycardia and sudden cardiac death $[10,11]$. However, the Tp-e and QT intervals might be affected by variations of body mass index (BMI) and heart rate (HR) [12]. Recently a new index, the Tp-e/QT ratio, has been suggested to be a more accurate measure of dispersion of ventricular repolarization compared to QT and Tp-e intervals, and to be independent of alterations in HR $[12,13]$.

Although ventricular repolarization was previously evaluated using QT dynamicity and heart rate variability (HRV) in patients with SSc [14], the novel repolarization indices Tp-e interval and $\mathrm{Tp}$-e/QT ratio have not been studied in SSc patients before. Therefore, the present study aimed to evaluate repolarization dispersion by using Tp-e interval and Tp-e/QT ratio in patients with systemic sclerosis.

\section{Methods}

\section{Study population}

A total of 149 patients with SSc who fulfilled the American College of Rheumatology criteria [15] for diagnosis of SSc and presented to the outpatient clinic of cardiology and rheumatology departments, were enrolled in this study. All subjects had a complete history and physical examination. Basic demographic characteristics including age, gender, BMI, systolic and diastolic blood pressure (BP) and HR of the whole population were recorded. Patients with SSc were screened in terms of disease duration (defined as interval between date of diagnosis and inclusion into study), and presence of digital ulcer [16], Raynaud's phenomenon [17] and severity of skin involvement in SSc patients were determined by the modified Rodnan method and modified Rodnan skin severity score (MR-SSS) calculated for all SSc patients. At more detailed method description for MR-SSS can be found in a study reported by Czirjak et al. [18].

Patients with diabetes mellitus, hypertension, dyslipidemia, chronic renal failure, chronic liver disease, neurologic diseases, known coronary artery or structural heart disease, pulmonary hypertension, rhythm abnormalities, use of drugs having effects on cardiac repolarization such as antiarrhythmic medications and smokers were excluded from the study. Patients with bundle branch block, atrioventricular conduction abnormalities on ECG and ECGs without clearly analyzable QT and $\mathrm{Tp}$-e intervals were also excluded. After taking into account these exclusion criteria, a total of $107 \mathrm{SSc}$ patients were found suitable and included in our study. During the enrollment process, 3 patients with pulmonary hypertension, 2 patients with neurological diseases and 2 patients with coronary artery disease were excluded. A total of 100 volunteers matched for age and sex with no previous history of cardiac disease served as the control group. All patients were in New York Heart Association (NYHA) class I. Complete blood count and biochemical markers in whole population were analyzed. Further, all patients were screened for the following immunological markers: anti-nuclear antibodies (ANA), anti-centromere antibodies (ACA), anti-cardiolipin IgG and IgM, anti-phospholipid IgG and IgM, anti-extractable nuclear antigen antibodies complex (ENA, SSA/Ro, SSB/La, Smith [Sm], ribonucleoprotein [RNP]), anti-topoisomerase I (anti-Scl-70), anti-Jo-1 and anti-double stranded DNA (anti-ds-DNA) antibodies.

The study was approved by the local ethics committee, and patients gave informed written consent. 


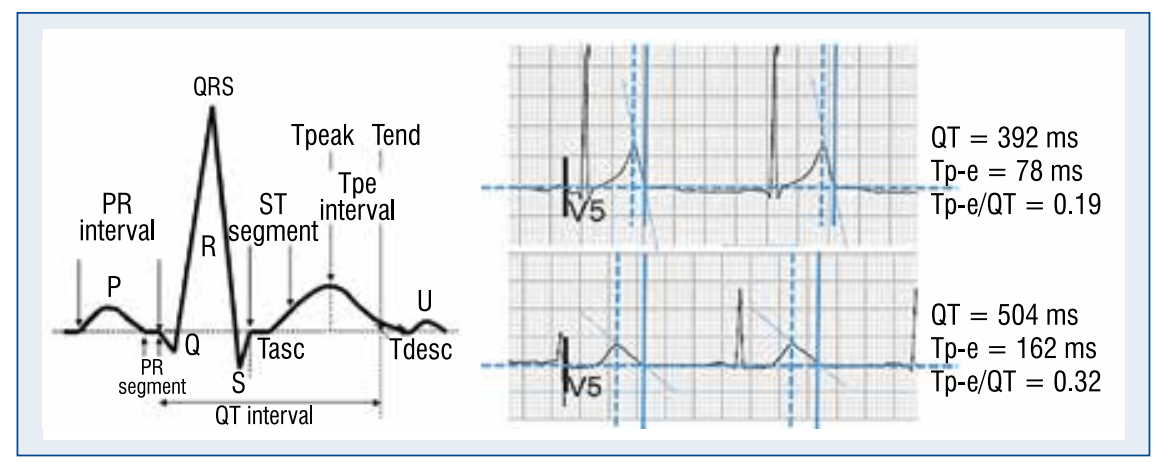

Figure 1. Measurements of Tp-e interval and the Tp-e/QT ratio. Please note the marked difference between a patient with systemic sclerosis and the control group.

\section{Transthoracic echocardiography}

All patients underwent transthoracic echocardiography. Echocardiographic examination was performed in the left lateral position from parasternal long- and short-axes, and apical 2- and 4 -chamber views. From the parasternal long axis, the left ventricular (LV) end-diastolic diameter and the LV end-systolic diameter were measured using M-mode (at the mitral chordal level perpendicular to the long axis of the ventricle). Left ventricular ejection fraction was calculated from apical 4-chamber view, according to the modified Simpson's rule [19]. The measurements were based on the criteria proposed by the American Society of Echocardiography [20]. In addition, left atrial diameter in parasternal long axis view and right ventricular end-diastolic diameter in apical 4-chamber view were also calculated. Pulmonary systolic arterial pressure was estimated by continuous wave (CW) Doppler as peak tricuspid regurgitation gradient plus an assumed right atrial pressure in relation to the size and respiratory variation of inferior vena cava visualized in subcostal view [19]. Measurements of mitral inflow included the peak early filling (E-wave) and late diastolic filling (A-wave) velocities, deceleration time of early filling velocity, and the isovolumic relaxation time, derived by placing the cursor of the CW Doppler in the LV outflow tract to simultaneously display the end of aortic ejection and the onset of mitral inflow. Pericardial effusion was determined by echocardiography and recorded if present.

\section{Electrocardiogram and measurement of indices}

The 12-lead ECG was recorded at a paper speed of $50 \mathrm{~mm} / \mathrm{s}$ (Nihon Kohden, Tokyo, Japan) at rest in the supine position. An "appropriate" ECG was defined as at least 10 analyzable leads for the required measurements. Otherwise, the ECG was considered noisy. Resting HR was measured from the ECG taken during the patient evaluation. ECG measurements of QT and Tp-e intervals were performed by a cardiologist who was blinded to the patient data and verified by a second physician to avoid error in measurements. An average value of 3 readings was calculated for each lead. The QT interval was measured from the onset of the QRS complex to the point at which the tangent of the maximal downslope of the descending limb of the $T$ wave crossed the isoelectric baseline and was corrected for HR using the Bazett's formula: QTc $=\mathrm{QT} / \sqrt{ } \mathrm{R}-\mathrm{R}$ interval [21]. The Tp-e interval was defined as the interval from the peak of $\mathrm{T}$ wave to the end of T wave. Measurements of Tp-e interval were performed from precordial leads [22]. Finally, the Tp-e/QT ratio was calculated from these measurements (Fig. 1).

All ECG measurements were performed by 2 independent cardiologists who were blinded to other patient information (mean of 2 measurements were used for analysis). Intra-observer agreement for QT and Tpe measurements was substantial (for QT measurement, simple agreement was $96 \%$ and Kappa (K): 0.86-0.95, p = 0.001; for Tp-e measurement, simple agreement was $94 \%$ and K: $0.84-0.93$, $\mathrm{p}=0.001)$. Similarly, inter-observer agreement was also satisfactory (for QT measurement, simple agreement was $94 \%$, K: 0.84-0.88, $\mathrm{p}=0.001$; for Tp-e measurement, simple agreement was $92 \%$, $\mathrm{K}: 0.70-0.82, \mathrm{p}=0.001$ ).

\section{Statistical analyses}

Statistical analyses were performed using SPSS for Windows 20 (SPSS Inc., Chicago, IL, USA). Numerical variables with a normal distribution were presented as mean \pm standard deviation, 
Table 1. Baseline demographic characteristics and echocardiographic parameters of the two groups.

\begin{tabular}{lccc}
\hline Variable & Systemic sclerosis group $(\mathbf{n}=\mathbf{1 0 7})$ & Control group $(\mathbf{n}=100)$ & $\mathbf{P}$ \\
\hline Age [years] & $48.6 \pm 14.0$ & $49.4 \pm 8.6$ & 0.613 \\
Gender (female/male) & $96 / 11$ & $90 / 10$ & 0.947 \\
Basal heart rate [min] & $72.1 \pm 11.1$ & $73.0 \pm 9.8$ & 0.547 \\
Body mass index [kg/m ${ }^{2}$ ] & $22.3 \pm 4.3$ & $23.1 \pm 5.4$ & 0.238 \\
Systolic BP [mm Hg] & $120.7 \pm 12.7$ & $118.3 \pm 11.5$ & 0.162 \\
Diastolic BP [mm Hg] & $71.4 \pm 7.9$ & $70.5 \pm 7.9$ & 0.403 \\
LV end-diastolic diameter [cm] & $4.53 \pm 0.32$ & $4.60 \pm 0.28$ & 0.102 \\
LV end-systolic diameter [cm] & $2.86 \pm 0.29$ & $2.79 \pm 0.29$ & 0.096 \\
LVEF [\%] & $64.1 \pm 3.5$ & $64.7 \pm 2.7$ & 0.167 \\
Left atrial dimension [cm] & $3.29 \pm 0.35$ & $3.36 \pm 0.35$ & 0.134 \\
RV end-diastolic diameter [cm] & $2.56 \pm 0.21$ & $2.53 \pm 0.24$ & 0.368 \\
Systolic PAP [mm Hg] & $27.5 \pm 4.9$ & $26.3 \pm 5.5$ & 0.098 \\
E wave [cm/s] & $79.6 \pm 14.9$ & $82.5 \pm 15.4$ & 0.170 \\
A wave [cm/s] & $57.1 \pm 15.3$ & $54.4 \pm 14.9$ & 0.200 \\
Deceleration time [ms] & $176.7 \pm 42.3$ & $183.0 \pm 45.7$ & 0.304 \\
Isovolumic relaxation time [ms] & $91.8 \pm 13.7$ & $88.5 \pm 14.8$ & 0.097 \\
MR-SSS & $19.2 \pm 6.2$ & $\mathrm{NA}$ & \\
\hline
\end{tabular}

Numerical variables with a normal distribution were presented as mean \pm standard deviation and those without a normal distribution were presented as median [minimum - maximum]; BP — blood pressure; LV — left ventricle; LVEF — left ventricular ejection fraction; RV — right ventricle; PAP — pulmonary arterial pressure; MR-SSS — modified Rodnan skin sclerosis score

numerical variables with a skewed distribution were presented as median [minimum and maximum] and categorical variables were presented as percentages. For numerical variables, an independent sample t-test and Mann-Whitney U test were used for inter-group comparisons. Pearson $\chi^{2}$ test was used to compare categorical variables. Pearson correlation analysis was used to test normal variables, while Spearman correlation analysis was used for variables not showing normal distribution. Multiple linear regression analysis was used to determine possible predictors among risk factors. $\mathrm{P}<0.05$ was considered statistically significant.

\section{Results}

\section{General characteristics of study population}

Baseline demographic characteristics and echocardiographic parameters were similar between the two groups. These parameters are shown in Table 1. Pericardial effusion was seen in none of the controls, while mild pericardial effusion $(<10 \mathrm{~mm})$ was seen in $23.4 \%(\mathrm{n}=25)$ of the SSc patients. The median disease duration was 76 months (minimum 1 month, maximum 480 months) in the SSc group. Complete blood count and biochemistry values of the two groups are shown in Table 2 . There were no statistically significant differences between the groups in terms of these laboratory parameters. A digital ulcer was present in $54.2 \%(\mathrm{n}=58)$ and Raynaud's phenomenon was present in $50.5 \%(\mathrm{n}=54)$ of SSc patients. The main clinical and serological data of SSc patients are represented in Table 3.

\section{Electrocardiographic indices}

Tp-e interval $(90.7 \pm 23.8 \mathrm{~ms}$ vs. $84.0 \pm 20.6 \mathrm{~ms}$, $\mathrm{p}=0.032$, respectively) and Tp-e/QT ratio $(0.20 \pm$ \pm 0.05 vs. $0.18 \pm 0.04, p=0.007$, respectively) were significantly higher in SSc patients when compared to those of the control group. However, QTc interval $(422 \pm 24 \mathrm{~ms}$ vs. $426 \pm 31 \mathrm{~ms}, \mathrm{p}=$ $=0.365$ ) was found to be similar in SSc patients with respect to the control group (Fig. 2).

Effects of all independent variables on Tp-e interval were examined separately in a univariate linear regression analysis. Erythrocyte sedimentation rate $(\mathrm{ESR})(\mathrm{p}=0.001, \beta=0.576)$, C-reactive protein $(\mathrm{CRP} ; \mathrm{p}=0.001, \beta=4.075)$, and MR-SSS ( $\mathrm{p}=0.001$, $\beta=2.373$ ) were found to be significant predictors of Tp-e interval. These significant variables were further analyzed in a multivariate linear regression model. MR-SSS $(\beta=2.108,95 \%$ CI 1.528-2.688, $\mathrm{p}=0.001)$ and CRP $(\beta=2.273,95 \%$ CI 0.258 $-4.287, \mathrm{p}=0.027$ ) were found to be significant independent predictors of Tp-e interval. 
Table 2. Complete blood count and biochemistry values of the two groups.

\begin{tabular}{lccc}
\hline Variable & Systemic sclerosis group $(\mathbf{n}=107)$ & Control group $(\mathbf{n}=100)$ & $\mathbf{P}$ \\
\hline Hemoglobin $[\mathrm{g} / \mathrm{dL}]$ & $12.4 \pm 1.4$ & $12.7 \pm 2.0$ & 0.210 \\
White blood cell count $[/ \mu \mathrm{L}]$ & $6700[5100-10000]$ & $6350[3000-12000]$ & 0.233 \\
Platelet count $[/ \mu \mathrm{L}]$ & $251000 \pm 99000$ & $236000 \pm 87000$ & 0.249 \\
Creatinine $[\mathrm{mg} / \mathrm{dL}]$ & $0.74 \pm 0.17$ & $0.79 \pm 0.22$ & 0.067 \\
Blood urea nitrogen $[\mathrm{mg} / \mathrm{dL}]$ & $17.1 \pm 4.1$ & $16.5 \pm 3.4$ & 0.254 \\
LDL-cholesterol $[\mathrm{mg} / \mathrm{dL}]$ & $119.4 \pm 41.4$ & $115.6 \pm 42.7$ & 0.516 \\
HDL-cholesterol $[\mathrm{mg} / \mathrm{dL}]$ & $43.5 \pm 11.2$ & $41.6 \pm 9.8$ & 0.196 \\
Triglyceride $[\mathrm{mg} / \mathrm{dL}]$ & $190.3 \pm 58.8$ & $185.6 \pm 60.1$ & 0.570 \\
Erythrocyte sedimentation rate $[\mathrm{mm} / \mathrm{h}]$ & $16[2-90]$ & $15[2-56]$ & 0.035 \\
C-reactive protein $[\mathrm{mg} / \mathrm{dL}]$ & $0.5[0.01-14.6]$ & $0.4[0.01-4.7]$ & 0.056 \\
Procalcitonin $[\mathrm{ng} / \mathrm{mL}]$ & $1.1[0.01-9.0]$ & $1.0[0.03-5]$ & 0.239 \\
Alanine aminotransferase $[\mathrm{U} / \mathrm{L}]$ & $22.3 \pm 6.7$ & $24.1 \pm 8.6$ & 0.093 \\
Aspartate aminotransferase $[\mathrm{U} / \mathrm{L}]$ & $29.5 \pm 9.6$ & $28.1 \pm 9.1$ & 0.283 \\
\hline
\end{tabular}

Numerical variables with normal distribution were presented as the mean \pm standard deviation and those without normal distribution were presented as median [minimum - maximum]; LDL — low density lipoprotein; HDL — high density lipoprotein cholesterol

Table 3. Main clinical and serologic data of systemic sclerosis patients.

\begin{tabular}{lc}
\hline Variable & $\mathrm{N}(\%)$ \\
\hline Digital ulcer positivity & $58(54.2 \%)$ \\
Raynaud's phenomenon positivity & $54(50.5 \%)$ \\
Anti-nuclear antibodies positivity & $82(76.6 \%)$ \\
Anti-double stranded DNA antibodies & $10(9.3 \%)$ \\
positivity & \\
Anti-centromere antibodies positivity & $8(7.5 \%)$ \\
Anti-extractable nuclear antigen & $61(57.0 \%)$ \\
antibodies positivity & \\
Anti-topoisomerase I (anti-Scl-70) & $48(44.9 \%)$ \\
antibodies positivity & $8(7.5 \%)$ \\
Anti SSA/Ro antibodies positivity & $0(0 \%)$ \\
Anti SSB/La antibodies positivity & $1(0.9 \%)$ \\
Anti Smith (Sm) antibodies positivity & $12(11.2 \%)$ \\
Anti ribonucleoprotein (RNP) & \\
antibodies positivity & $0(0 \%)$ \\
Anti-Jo-1 antibodies positivity & $4(3.7 \%)$ \\
Anti-cardiolipin IgM antibodies & \\
positivity & $5(4.7 \%)$ \\
Anti-cardiolipin IgG antibodies & $1(0.9 \%)$ \\
positivity & $2(1.9 \%)$ \\
Anti-phospholipid IgM antibodies & \\
positivity & Anti-phospholipid IgG antibodies \\
positivity & \\
\hline
\end{tabular}

Similarly, influence of all independent variables on Tp-e/QT ratio was examined separately in a univariate linear regression analysis. ESR $(\mathrm{p}=0.001, \beta=0.001), \mathrm{CRP}(\mathrm{p}=0.001, \beta=0.008)$, and MR-SSS ( $\mathrm{p}=0.001, \beta=0.005)$ were found to be significant predictors of $\mathrm{Tp}-\mathrm{e} / \mathrm{QT}$ ratio. These significant variables were further analyzed in multivariate linear regression model. MR-SSS $\beta=0.004$, $95 \%$ CI $0.003-0.003, \mathrm{p}=0.001$ ) was found to be only significant independent predictors of Tp-e/QT ratio among patients with SSc.

Pearson's correlation analyses revealed positive correlations of MR-SSS with QTc $(r=0.427$, $\mathrm{p}=0.001)$, Tp-e interval $(\mathrm{r}=0.620, \mathrm{p}=0.001)$ and Tp-e/QT ratio $(\mathrm{r}=0.615, \mathrm{p}=0.001)$ (Fig. 3). There were also weak positive correlations of ESR with Tp-e interval $(\mathrm{r}=0.328, \mathrm{p}=0.001)$ and Tp-e/ $/ \mathrm{QT}$ ratio $(\mathrm{r}=0.308, \mathrm{p}=0.001)$.

\section{Discussion}

The main findings of the present study are as follows: (1) repolarization dispersion assessed by using Tp-e interval and $\mathrm{Tp}$-e/QT ratio was prolonged in patients with SSc when compared to that of the controls, (2) this prolongation was well correlated with MR-SSS, (3) MR-SSS was found to be an independent predictor of both Tp-e interval and $\mathrm{Tp}-\mathrm{e} / \mathrm{QT}$ ratio.

To the best of our knowledge, this is the first study to report on Tp-e interval and Tp-e/QT ratio in SSc patients. The Tp-e/QT ratio is a relatively new index of increased dispersion of ventricular repolarization and is considered to be a more sensitive index of arrhythmogenesis compared to sole use of either Tp-e or QT intervals [12]. Therefore, 


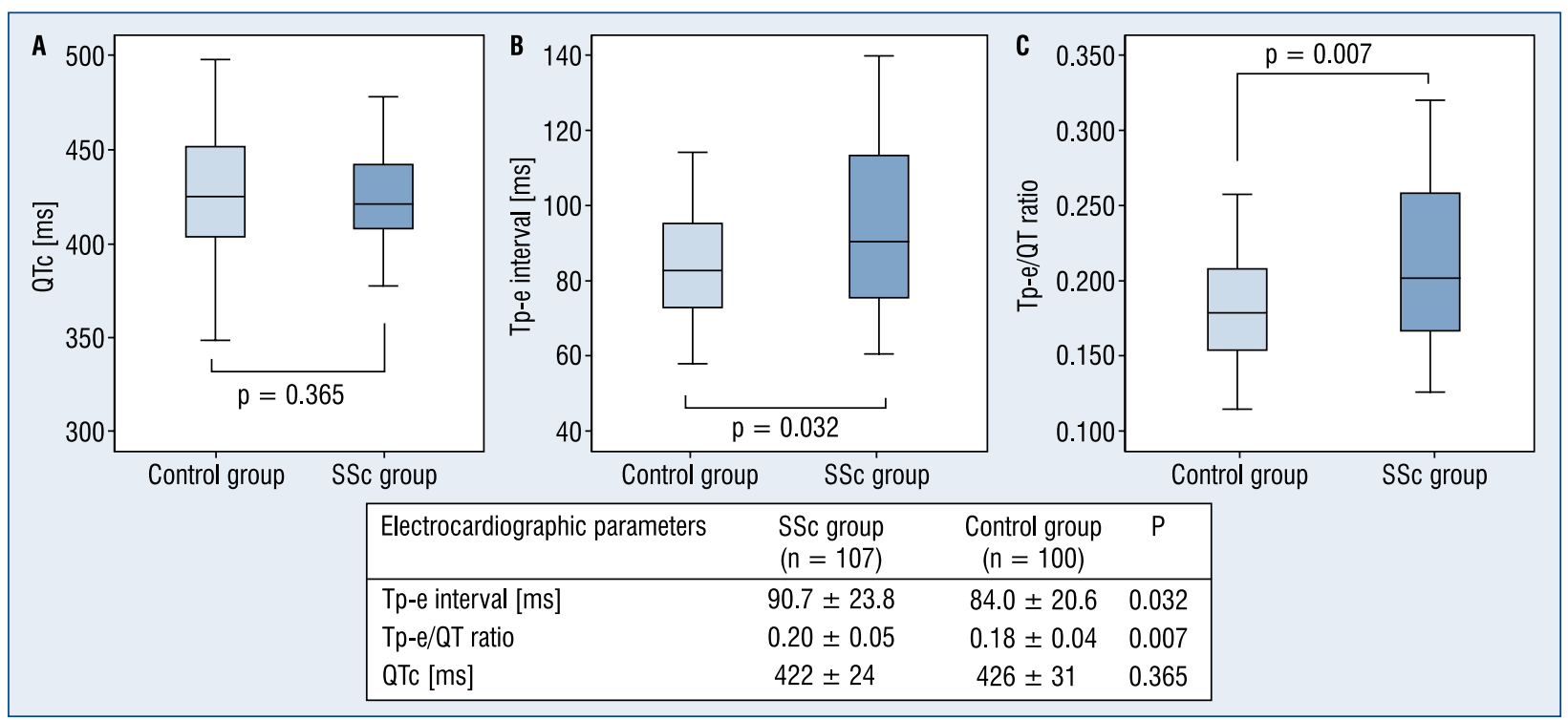

Figure 2. QTc (A), Tp-e interval (B) and Tp-e/QT ratio (C) in patients with systemic sclerosis (SSc) and the control group.

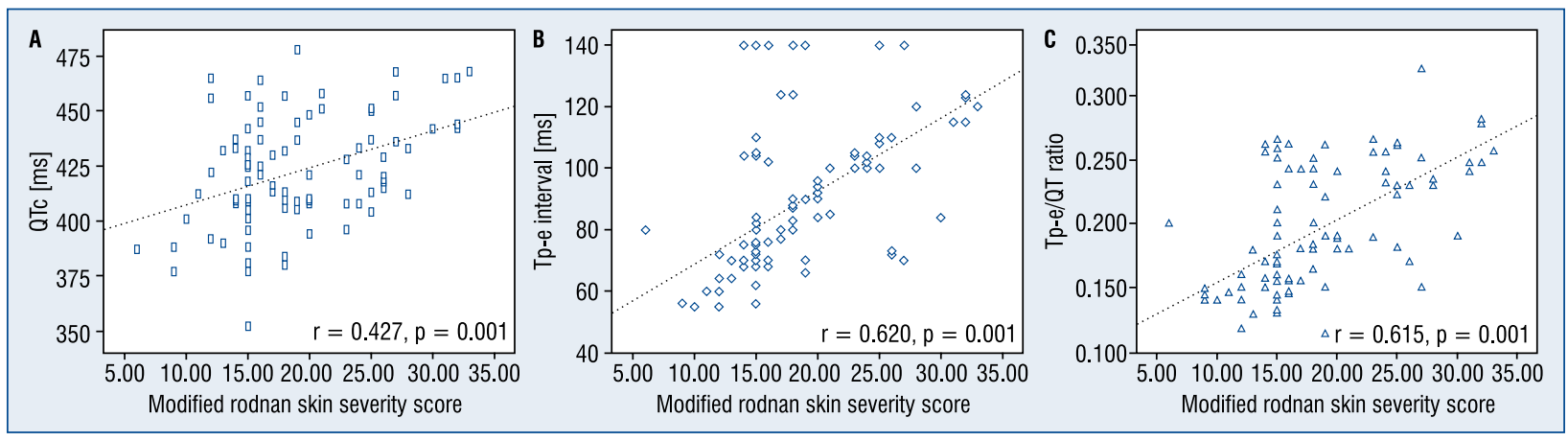

Figure 3. Correlation of Modified Rodnan skin severity score with (A) QTc, (B) Tp-e interval and (C) Tp-e/QT ratio; $r$ indicates correlation coefficient.

this ratio is being used increasingly more frequently as an electrocardiographic index of ventricular arrhythmogenesis in various diseases. Previous studies have shown that prolongation of the Tp-e interval is associated with increased mortality in Brugada syndrome, hypertrophic cardiomyopathy, and in patients undergoing primary percutaneous coronary intervention for myocardial infarction [13, 22, 23]. Yamaguchi et al. [24] found that the Tp-e/QT ratio is a superior predictor of torsades de pointes than either QTc interval or QTd in patients with long QT syndrome (LQTS). Likewise, QTc did not reach statistical significance between the two groups in our study. However, Tp-e interval and Tp-e/QT ratio were prolonged in patients with SSc when compared to that of the controls.
Many studies have evaluated abnormalities of the ventricular myocardium and arrhythmias and markers of such arrhythmias in patients with SSc. It has been found that SSc patients show tendency to tachycardia and have significantly lower heart rate turbulence values compared to controls [25, 26]. Similarly, Çiftçi et al. [14], using 24-h ambulatory ECG recording, found that diffuse $\mathrm{SSc}$ is associated with a significant worsening of HRV and QT dynamicity parameters. In another study, Paradiso et al. [27], using signal averaged ECG, found that nearly half of SSc patients have late ventricular potentials. Additionally, Sgreccia et al. [28] reported that patients with SSc have increased QTc interval, QTd, and QTc dispersion.

Various mechanisms have been proposed for heterogeneity in ventricular repolarization and 
abnormalities in markers of arrhythmogenesis in patients with SSc. The first possible mechanism is inflammation and fibrosis of cardiac tissues. Autoimmunity, vasculopathy and fibrosis, which are the basic components of pathophysiology in SSc, affect the heartjust as they affect all organ systems [3]. Cardiac vascular involvement in SSc is usually seen in small arterial segments and exerciseinduced perfusion defects despite normal coronary angiogram are thought to be related to vasospasm and abnormal resistance to flow at the level of the microcirculation or myocardial interstitium [29]. Fibrotic process can occur directly due to inflammatory and autoimmune mechanisms and it may also be ischemia-mediated as a result of abnormality in microcirculation [5]. This fibrotic process that is seen in SSc tends to be patchy $[30,31]$. In addition, it is well recognized that ventricular myocardium is electrically a heterogeneous structure [32]. The cells located in the subendocardial layer have the longest action potential duration followed by endocardial and epicardial cells [12]. Therefore, transmural dispersion caused by this voltage gradient between layers may be damaged by a fibrotic process similar to that in SSc. In our study, supporting data above, higher Tp-e interval and Tp-e/QT ratio was well correlated with MR-SSS, and MR-SSS was found to be an independent predictor of both Tp-e interval and Tp-e/QT ratio.

The second possible mechanism is dysregulation of the autonomic nervous system. Vaseghi et al. [33] found that sympathetic stimulation increases Tp-e interval and thus myocardial dispersion of repolarization. Additionally, Tanabe et al. [34] showed that sympathetic stimulation produces a greater increase in both transmural and spatial dispersion of repolarization in patients with LQTS. In light of these studies, similar autonomic dysregulation which could be seen in SSc may increase dispersion of repolarization and predispose to ventricular arrhythmias [35].

In the present study, there were weak significant positive correlations of Tp-e interval and Tp-e/ /QT ratio with ESR. Furthermore, CRP was found to be another independent predictor of Tp-e interval. Inflammation is known to play an essential role in the pathogenesis of cardiovascular diseases such as coronary artery disease and arrhythmias [36]. This finding supports the above-mentioned effect of inflammatory mechanisms on the ventricular myocardium and thus, supports the tendency of arrhythmia seen in an inflammatory disease, such as SSc. This relationship between inflammatory activity and $\mathrm{Tp}$-e interval and $\mathrm{Tp}$-e/QT ratio is similar to the result of studies performed on patients with rheumatic arthritis and ankylosing spondylitis [37, 38].

Our results may contribute to the pathophysiological mechanisms of increased prevalence of ventricular arrhythmias and increased cardiovascular mortality risk by indicating increased ventricular repolarization heterogeneity and increased inflammation in these patients. The increased frequency of ventricular arrhythmias and sudden cardiac death might be explained by prolonged transmural dispersion and chronic ongoing systemic inflammation in SSc patients. Therefore, our findings may be a reference for further studies.

\section{Limitations of the study}

The major limitation of our study is its crosssectional design and lack of follow-up of the patients. The number of patients included is very small and since we did not assess the association between ventricular arrhythmias with Tp-e interval and $\mathrm{Tp}-\mathrm{e} / \mathrm{QT}$ ratio, there is no information regarding any cardiovascular endpoints. Therefore, we could not evaluate the potential prognostic role of the electrocardiographic ventricular repolarization indices with respect to future events. In regards to patients included in study, organ complications might preclude participation. Therefore, study population might be skewed to the early phases of the disease. Additionally, since a very limited group of patients were included due to several exclusion criteria, and since no distinction was made between limited/diffuse SSc, the results of this study cannot be generalized to the whole SSc patient population. For this reason, long-term follow-up and large-scale prospective studies are needed to determine the predictive value of prolonged Tp-e interval and increased Tp-e/QT ratio, and to understand the pathophysiology, as well as to make accurate clinical decisions in this population.

\section{Conclusions}

Our study pointed out that Tp-e interval and Tp-e/QT ratio were increased in SSc patients. Our results also showed that these electrocardiographic indices of ventricular repolarization were significantly correlated with MR-SSS. Furthermore, MR-SSS was found to be a significant independent predictor of Tp-e interval and Tp-e/QT ratio among patients with SSc. When the prognostic significance of Tp-e interval and Tp-e/QT ratio is considered, patients with SSc, particularly with higher MR-SSS, should be followed closely for 
adverse cardiovascular outcomes. Further studies are required to understand the pathogenesis and consequences of repolarization dispersion and ventricular arrhythmias in systemic sclerosis.

\section{Conflict of interest: None declared}

\section{References}

1. Jimenez SA, Derk CT. Following the molecular pathways toward an understanding of the pathogenesis of systemic sclerosis. Ann Internal Med, 2004; 140: 37-50.

2. LeRoy EC, Black C, Fleischmajer R et al. Scleroderma (systemic sclerosis): Classification, subsets and pathogenesis. J Rheumatol, 1988; 15: 202-205.

3. Champion HC. The heart in scleroderma. Rheumatic Diseases Clinics of North America, 2008; 34: 181-190; viii.

4. Morelli S, Sgreccia A, Ferrante L et al. Relationships between electrocardiographic and echocardiographic findings in systemic sclerosis (scleroderma). Int J Cardiol, 1996; 57: 151-160.

5. Steen V. The heart in systemic sclerosis. Current Rheumatology Reports, 2004; 6: 137-140.

6. Antzelevitch C. Heterogeneity and cardiac arrhythmias: An overview. Heart Rhythm, 2007; 4: 964-972.

7. Yan GX, Martin J. Electrocardiographic T wave: A symbol of transmural dispersion of repolarization in the ventricles. J Cardiovasc Electrophysiol, 2003; 14: 639-640.

8. Antzelevitch C, Sicouri S, Di Diego JM et al. Does Tpeak-Tend provide an index of transmural dispersion of repolarization? Heart Rhythm, 2007; 4: 1114-1116; author reply 1116-1119.

9. Panikkath R, Reinier K, Uy-Evanado A et al. Prolonged Tpeakto-tend interval on the resting ECG is associated with increased risk of sudden cardiac death. Circ Arrhythm Electrophysiol, 2011; 4: 441-447.

10. Moss AJ, Schwartz PJ, Crampton RS, Locati E, Carleen E. The long QT syndrome: A prospective international study. Circulation, 1985; 71: 17-21.

11. Bjerregaard P, Gussak I. Short QT syndrome. Ann Noninvasive Electrocardiol, 2005; 10: 436-440.

12. Gupta P, Patel C, Patel H et al. T(p-e)/QT ratio as an index of arrhythmogenesis. J Electrocardiol, 2008; 41: 567-574.

13. Zhao X, Xie Z, Chu Y et al. Association between Tp-e/QT ratio and prognosis in patients undergoing primary percutaneous coronary intervention for ST-segment elevation myocardial infarction. Clin Cardiol, 2012; 35: 559-564.

14. Ciftci O, Onat AM, Yavuz B et al. Cardiac repolarization abnormalities and increased sympathetic activity in scleroderma. J National Med Association, 2007; 99: 232-237.

15. Preliminary criteria for the classification of systemic sclerosis (scleroderma). Subcommittee for scleroderma criteria of the American Rheumatism Association Diagnostic and Therapeutic Criteria Committee. Arthritis Rheumatism, 1980; 23: 581-590.

16. Amanzi L, Braschi F, Fiori $G$ et al. Digital ulcers in scleroderma: Staging, characteristics and sub-setting through observation of 1614 digital lesions. Rheumatology (Oxford), 2010; 49: 1374-1382.

17. Maverakis E, Patel F, Kronenberg DG et al. International consensus criteria for the diagnosis of Raynaud's phenomenon. J Autoimmunity, 2014; 48-49: 60-65.

18. Czirjak L, Foeldvari I, Muller-Ladner U. Skin involvement in systemic sclerosis. Rheumatology (Oxford), 2008; 47 (suppl. 5): v44-v45.

19. Lang RM, Bierig M, Devereux RB et al. Recommendations for chamber quantification: A report from the American Society of Echocardiography's Guidelines and Standards Committee and the Chamber Quantification Writing Group, developed in conjunction with the European Association of Echocardiography, a branch of the European Society of Cardiology. J Am Soc Echocardiogr, 2005; 18: 1440-1463.

20. Lang RM, Badano LP, Mor-Avi V et al. Recommendations for cardiac chamber quantification by echocardiography in adults: An update from the American Society of Echocardiography and the European Association of Cardiovascular Imaging. Eur Heart J Cardiovasc Imag, 2015; 16: 233-270.

21. Funck-Brentano C, Jaillon P. Rate-corrected QT interval: Techniques and limitations. Am J Cardiol, 1993; 72: 17B-22B.

22. Castro Hevia J, Antzelevitch C, Tornes Barzaga F et al. TpeakTend and Tpeak-Tend dispersion as risk factors for ventricular tachycardia/ventricular fibrillation in patients with the Brugada syndrome. J Am Coll Cardiol, 2006; 47: 1828-1834.

23. Shimizu M, Ino H, Okeie K et al. T-peak to T-end interval may be a better predictor of high-risk patients with hypertrophic cardiomyopathy associated with a cardiac troponin I mutation than QT dispersion. Clin Cardiol, 2002; 25: 335-339.

24. Yamaguchi M, Shimizu M, Ino $\mathrm{H}$ et al. T wave peak-to-end interval and QT dispersion in acquired long QT syndrome: A new index for arrhythmogenicity. Clinical Science, 2003; 105: 671-676.

25. Wranicz JK, Strzondala M, Zielinska M et al. [Evaluation of early cardiovascular involvement in patients with systemic sclerosis]. Przegl Lek, 2000; 57: 389-392.

26. Bienias P, Ciurzynski M, Glinska-Wielochowska $M$ et al. Heart rate turbulence impairment and ventricular arrhythmias in patients with systemic sclerosis. Pacing Clin Electrophysiol, 2010; 33: 920-928.

27. Paradiso M, Di Franco M, Musca A et al. Ventricular late potentials in systemic sclerosis: Relationship with skin involvement. J Rheumatol, 2002; 29: 1388-1392.

28. Sgreccia A, Morelli S, Ferrante L et al. QT interval and QT dispersion in systemic sclerosis (scleroderma). J Internal Med, 1998; 243: 127-132.

29. Follansbee WP, Curtiss EI, Medsger TA, Jr., Owens GR, Steen VD, Rodnan GP. Myocardial function and perfusion in the CREST syndrome variant of progressive systemic sclerosis. Exercise radionuclide evaluation and comparison with diffuse scleroderma. Am J Med, 1984; 77: 489-496.

30. Deswal A, Follansbee WP. Cardiac involvement in scleroderma. Rheumatic diseases Clinics of North America, 1996; 22: 841-860.

31. Bulkley BH, Ridolfi RL, Salyer WR, Hutchins GM. Myocardial lesions of progressive systemic sclerosis. A cause of cardiac dysfunction. Circulation, 1976; 53: 483-490.

32. Sicouri S, Antzelevitch C. A subpopulation of cells with unique electrophysiological properties in the deep subepicardium of the canine ventricle. The M cell. Circ Res, 1991; 68: 1729-1741.

33. Vaseghi M, Yamakawa K, Sinha A et al. Modulation of regional dispersion of repolarization and T-peak to T-end interval by the right and left stellate ganglia. Am J Physiol Heart Circ Physiol, 2013; 305: H1020-H1030.

34. Tanabe Y, Inagaki M, Kurita $\mathrm{T}$ et al. Sympathetic stimulation produces a greater increase in both transmural and spatial dispersion of repolarization in LQT1 than LQT2 forms of congenital long QT syndrome. J Am Coll Cardiol, 2001; 37: 911-919.

35. Karakulak UN, Okutucu S, Sahiner L et al. Assessment of cardiac autonomic nervous system involvement in systemic sclerosis via exercise heart rate recovery. Med Princ Pract 2015; 24: $17-22$.

36. Mountantonakis S, Deo R. Biomarkers in atrial fibrillation, ventricular arrhythmias, and sudden cardiac death. Cardiovascular Therapeutics, 2012; 30: e74-e80.

37. Acar GR, Akkoyun M, Nacar AB et al. Evaluation of Tp-e interval and $\mathrm{Tp}-\mathrm{e} / \mathrm{QT}$ ratio in patients with rheumatoid arthritis. Turk Kardiyol Dern Ars, 2014; 42: 29-34.

38. Acar G, Yorgun H, Inci MF et al. Evaluation of Tp-e interval and Tp-e/QT ratio in patients with ankylosing spondylitis. Modern Rheumatology/the Japan Rheumatism Association 2013. 